\title{
Filigrane
}

Écoutes psychanalytiques

\section{FtM et psychanalyse - hypothèses sur un silence théorique}

\section{Fanny Chevalier}

Volume 28, numéro 2, 2019

Identités $^{2}$. Qui suis-je ? Deuxième partie

URI : https://id.erudit.org/iderudit/1069694ar

DOI : https://doi.org/10.7202/1069694ar

Aller au sommaire du numéro

Éditeur(s)

Santé mentale et société

ISSN

1192-1412 (imprimé)

1911-4656 (numérique)

Découvrir la revue

Citer cet article

Chevalier, F. (2019). FtM et psychanalyse - hypothèses sur un silence théorique. Filigrane, 28(2), 45-69. https://doi.org/10.7202/1069694ar
Résumé de l'article

La littérature psychanalytique lacanienne concernant le " transsexualisme " manifeste un effacement significatif de la mention des transitions female-to-male (FtM) au profit d'un intérêt permanent et quasi exclusif pour les male-to-female (MtF) - au point alors de faire de ces derniers la figure du transsexualisme. Comment rendre compte du désintérêt psychanalytique à l'égard des FtM ? Dans quelle mesure est-il ordonné par les théories, freudiennes en particulier, sur la féminité ? L'étude de la place accordée au différentiel FtM/MtF dans les textes psychanalytiques montrera par ailleurs que les abords du transsexualisme semblent indissociables de considérations diagnostiques, ce qui conduira à interroger en dernier lieu la tendance psychanalytique à faire « du » transsexualisme une entité structurale. 


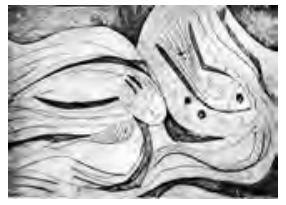

\title{
FtM et psychanalyse - hypothèses sur un silence théorique
}

\author{
Fanny Chevalier
}

\begin{abstract}
Résumé: La littérature psychanalytique lacanienne concernant le «transsexualisme» manifeste un effacement significatif de la mention des transitions femaleto-male (FtM) au profit d'un intérêt permanent et quasi exclusif pour les male-tofemale $(\mathrm{MtF})$ - au point alors de faire de ces derniers la figure du transsexualisme. Comment rendre compte du désintérêt psychanalytique à l'égard des FtM? Dans quelle mesure est-il ordonné par les théories, freudiennes en particulier, sur la féminité? L'étude de la place accordée au différentiel FtM/MtF dans les textes psychanalytiques montrera par ailleurs que les abords du transsexualisme semblent indissociables de considérations diagnostiques, ce qui conduira à interroger en dernier lieu la tendance psychanalytique à faire «du» transsexualisme une entité structurale.
\end{abstract}

Mots clés: FtM; MtF; transsexualisme féminin; féminité; masculinité; identité.

\begin{abstract}
A literature review of Lacanian approaches to "transsexualism" shows that female-to-male (FtM) transition is significantly overlooked and that psychoanalysis seems to be persistently and almost exclusively interested in male-to-female $(\mathrm{MtF})$ transition - to the extent that the latter can be said to have become the very epitome of transsexualism. How can the lack of psychoanalytical interest in FtM transition be addressed? To what extent is this structured around the Freudian theories, in particular those on femininity? The study of the place given to the differential between FtM/MtF in the psychoanalytic literature will also show that the questions surrounding transsexualism seem impossible to distinguish from diagnostic considerations, which will lead to the question of how psychoanalysis tends to think of "transsexualism" as one structural entity.
\end{abstract}

Keywords: FTM; MTF; feminine transsexualism; feminity, masculinity, identity.

"Transsexualisme féminin», "transsexuelles», et plus récemment «femmes transgenres»; c'est sous ces dénominations que la psychanalyse évoque les transitions female-to-male (FtM), là où le consensus social privilégie actuellement, tout à l'inverse, l'expression «hommes trans'». Même logique de renversement pour ce qui concerne les male-to-female $(\mathrm{MtF})$, que la psychanalyse nommera «transsexuels» ou qu'elle qualifiera 
de «transsexualisme masculin», quand la voix moderne les dira «femmes trans'». L'accent est donc fondamentalement porté sur des lieux différents, révélant d'emblée par là des logiques autres - ce qui dessine déjà une ligne de brisure dans l'étude de la question trans', selon qu'elle est abordée par la psychanalyse ou par un autre champ disciplinaire ${ }^{1}$.

\section{Du transsexualisme aux transidentités}

Ces soucis terminologiques, outre qu'ils n'affectent pas seulement les transitions dans leur aspect différentiel, manifestent à quel point le choix même de la dénomination est gros d'enjeux politiques et idéologiques. Le terme de «transsexualisme» est ainsi aujourd'hui sévèrement remis en cause en raison des connotations qu'il véhicule et tend ainsi à être considéré comme « une catégorie nosographique obsolète» (Alessandrin, 2012), dans l'intention d'arracher sa considération à une définition psychiatrique et à son «implicite pathologisant» (Alessandrin, 2012, p. 266). Les expressions «transidentités», «transgenre» ou «trans'» sont depuis largement privilégiées, sans que leur usage ne relève toutefois d'un consensus ferme. L'encyclopédie critique du genre considère en effet que le terme «transgenre», qui jusqu'à présent s'opposait au transsexualisme par la non-médicalisation du parcours, «désigne désormais la population non cisgenre dans son ensemble» (Beaubatie, 2016a, p. 643); par ailleurs, la préférence peut être accordée au seul préfixe indifférencié «trans' " plutôt qu'à la catégorie médicale de «transsexuel-le» ou à la catégorie militante de «transgenre» (Beaubatie, 2016a, p. 640). Françoise Sironi, quant à elle, définit la transidentité comme la catégorie générale pour désigner «l'ensemble des expériences de passage entre les genres», regroupant à ce titre «la transsexualité et les vécus transgenres», leur distinction mettant plutôt l'accent sur la binarité ou la non-binarité du genre, faisant des transgenres des «sujets déterritorialisés, à appartenances multiples», non "“assignés à résidence" dans un genre donné» (Sironi, 2011, p. 15-16).

Le délaissement du «transsexualisme» s'accorde donc sur la volonté de dépathologiser les vécus trans', tandis que le flottement qui demeure quant à son substitut légitime met précisément en exergue leur multiplicité, en lieu et place $d u$ transsexualisme tel qu'il était jusqu'alors circonscrit. La formule canonique qui définissait le transsexualisme comme une «erreur de la nature», et qui la constituait comme entité clinique - cette conviction d'être une femme prisonnière dans un corps d'homme, ou inversement s'en retrouve alors fortement ébranlée, proprement pulvérisée, comme en témoigne le pluriel qui est de mise lorsque sont évoquées les transidentités. 
Mais la transition de dénomination du transsexualisme aux transidentités ne procède pas de cette seule reprise du singulier au pluriel, qui met en relief la multiplicité des articulations entre image et semblant, corps, sexe, désir et nomination; elle se double d'une autre correction qui vise à rendre intelligible et manifeste l'idée selon laquelle le ressort en jeu ne relève pas du sexuel ou de la sexualité, mais bien de l'identité. Et c'est précisément cet effacement du sexuel au profit de la dimension identitaire qui n'est pas sans interroger tout particulièrement la psychanalyse.

«Le transsexualisme [...] ça n'est pas une question de sexe, mais d'identité» (Millot, 1983, p. 119); cet élément, déjà porté à la discussion en 1981, faisait dire à Marcel Czermak à la suite d'une intervention sur le transsexualisme du Dr Klotz, psychiatre, que «pour ce qui nous concerne, il est tout à fait exclu que nous puissions considérer que les faits de la sexualité soient distincts du phénomène de l'identité», dans la mesure où la sexualité entretient «le rapport le plus étroit avec la façon dont les phénomènes identificatoires ont opéré ou n'ont pas opéré pour tel ou tel sujet» (Klotz, 1981, p. 206). Cette promotion de la dimension identitaire désarrimée de toute référence à la sexualité ne peut que constituer une nouvelle fracture avec la psychanalyse sur le plan conceptuel, en ce sens que la sexualité ne saurait s'y laisser réduire à son exercice génital - ni même à des pratiques sexuelles qui se passeraient de la génitalité - ou encore seulement à une question de choix d'objet.

La littérature psychanalytique concernant ledit transsexualisme - puisque tel est le terme en vigueur dans le corpus travaillé ${ }^{2}$ - est de celles dont il est difficile d'établir le périmètre exact, quand bien même son étude se serait débarrassée de toute prétention à l'exhaustivité et qu'elle se restreindrait à une orientation théorique particulière - lacanienne ici ${ }^{3}$. Il n'en demeure pas moins qu'une remarque générale s'impose, rarement relevée et encore moins élevée au rang de questionnement: on constate en effet, sinon l'absence, du moins un effacement conséquent de la mention des transitions FtM au profit d'un intérêt permanent et quasi exclusif pour les $\mathrm{MtF}-\mathrm{au}$ point alors de faire des MtF la figure $d u$ transsexualisme. Sans que jamais ne soit pour autant niée leur existence, les FtM sont néanmoins laissés dans l'ombre, dans un désintérêt théorique qui ne manque pas d'interroger, tel un point aveugle qui se laisse difficilement circonscrire. 


\section{Invisibilité des FtM et mutisme académique}

La plus grande rareté des transitions FtM en regard des MtF a souvent été invoquée pour rendre compte de ce désintérêt. Ce motif a depuis été fermement révoqué, notamment par Julie Guillot et Emmanuel Beaubatie qui, avec ingéniosité, s'appuient non pas sur de nouveaux chiffres, mais bien sur un questionnement quant à la pertinence même de leur source ${ }^{4}$. Le constat préliminaire de ces sociologues confirme que l'absence de visibilité des MtF déborde largement le seul champ psychanalytique et se vérifie aussi bien «dans les médias, dans la littérature, dans les publications scientifiques, dans l'historiographie [que] dans les conceptions de sens commun» (Guillot et Beaubatie, 2012, p. 71). Pat Califia, essayiste transgenre, relevait déjà à l'occasion de son ouvrage Le mouvement transgenre, en 1997, que "les transsexuels FtM doivent se battre contre un vide culturel qui les rend invisibles » (Califia, 1997, p. 27). Que ce manque de visibilité soit aujourd'hui à relativiser - comme le soulignent les auteurs en conclusion de leur article - n'amende en rien l'intérêt de la démarche de Guillot et Beaubatie dont l'hypothèse principale revient, en quelque sorte, à substituer au seul constat d'invisibilité la notion d'invisibilisation. Ce néologisme a le mérite de mettre en exergue les processus qui œuvrent implicitement à ce "désintérêt académique, littéraire et médiatique» (Guillot et Beaubatie, 2012, p. 75), processus qui se déploient selon trois axes, évidemment intriqués. D’une part, l'androcentrisme scientifique, auquel on peut imputer la cause de ce silence historique quant aux FtM au même titre qu'il a affecté les femmes et les lesbiennes (Guillot et Beaubatie, 2012, p. 75). D’autre part, un déni portant sur l'existence même des hommes trans', entendus alors comme des homosexuelles refoulées ou des usurpatrices (Guillot et Beaubatie, 2012, p. 78); c'est à cet endroit d'ailleurs que la théorie psychanalytique est mentionnée - de façon quelque peu obscure, prise dans le mouvement confus des «disciplines psy» qui auraient participé à ces interprétations erronées à partir d'une théorie de la sexualité critiquable en ce qu'elle poserait l'homme comme enviable de fait. Mais l'argument majeur réside dans la mise à découvert de la force des résistances psychologiques qui s'élèvent contre l'idée même que la masculinité puisse être construite, autrement dit qu'elle ne soit pas naturellement liée à l'anatomie masculine: "l'existence des FtM, mais aussi des butchs, des femmes travesties, met en danger l'identité masculine en mettant en lumière son caractère construit, non naturel: en somme, si ce n'est plus la possession d'un pénis "naturel" qui définit ce qu'est un homme, alors qu'estce que c'est? [...] En d'autres termes: on nait homme, on ne le devient 
pas.» (Guillot et Beaubatie, 2012, p. 80 et 84) Ce caractère impensable de construction contribuerait à la non-visibilité des hommes trans', ceux-ci y participant d'ailleurs en adoptant les codes normatifs de la masculinité, en un consentement forcé.

L'objet de cet article consistera à discuter de la mesure dans laquelle cette hypothèse concernant le désintérêt théorique à l'égard des FtM conserve sa pertinence lorsqu'elle est appliquée au champ spécifique de la psychanalyse. Que ce silence soit mû par une obnubilation pour l'émasculation et donc une fascination par les transitions MtF, autrement dit, qu'il soit commandé par l'inscription de la psychanalyse dans une «logique hétéro-phallo-centriste» qu'elle ne se contenterait pas seulement de reproduire, mais d'entériner (Evzonas, 2019, p. 12) - cette hypothèse suffit-elle vraiment à clore ce questionnement sans de plus amples approfondissements?

S'il est besoin de le préciser, cet essai ne visera nullement à édifier une construction théorique qui rendrait spécifiquement compte des motifs inconscients poussant une femme à transitionner vers le genre masculin - visée dont l'entreprise trouvera d'ailleurs à être fermement critiquée quand elle est conçue selon une logique totalisante et unitaire qui prétendrait valoir pour tous les hommes trans'. Le propos est ailleurs et a bien pour objectif d'interroger la manière dont les théories psychanalytiques freudiennes et lacaniennes sur la féminité et la masculinité, même dans leurs aspects les plus critiqués historiquement, ordonnent silencieusement l'appréhension différentielle qui est faite des transidentités, jusqu'à pouvoir fonctionner comme des obturateurs. Il sera donc question de repérer les a priori théoriques et les systématismes propres à la psychanalyse qui concourent à faire des transitions FtM un point aveugle, un champ clinique qui ne recèlerait aucune énigme susceptible d'être explorée.

Il conviendra d'ailleurs dans un premier temps de relativiser l'absence d'intérêt de la psychanalyse pour les FtM, en ce qu'elle a évidemment connu des exceptions, et de présenter les caractéristiques qui distingueraient alors les transitions FtM des transitions MtF. Ce préliminaire permettra d'étudier plus largement la place qui est accordée au différentiel FtM/MtF et la manière dont celui-ci est traité dans les textes s'intéressant «au» transsexualisme, afin d'en arriver à l'hypothèse selon laquelle le triptyque MtFSchreber-psychose a tendance à fonctionner comme paradigme pour rendre compte $d u$ transsexualisme, quelles que soient ses déclinaisons. L'opérateur invoqué dans l'articulation de ce triptyque étant le "pousse-à-la-femme», expression forgée par Lacan en 1972, existe-t-il un concept équivalent du 
côté des transitions FtM? L’envie du pénis sera ici convoquée et resituée dans les débats historiques qui firent rage dans le monde psychanalytique, pour être confrontée aux transitions FtM; dans quelle mesure la résonance inattendue qui s'opère spontanément entre ces dernières et ce concept si controversé qu'est l'envie du pénis peut-elle être tenue pour responsable de la négligence psychanalytique à l'égard des FtM? Une dernière partie mettra en évidence d'une part que le traitement différentiel ou non des transitions est inséparable d'hypothèses concernant le diagnostic de structure et, d'autre part, que l'hypothèse de la névrose n'émerge jamais qu'en rapport aux FtM. Sera ainsi interrogée, en conclusion, la tendance psychanalytique à faire « du» transsexualisme une entité structurale.

\section{Spécificités du " transsexualisme féminin ${ }^{5}$ " en psychanalyse}

Les premières occurrences d'un intérêt d'orientation lacanienne pour les FtM datent de 1981, dans une série de courts articles parus dans la revue Ornicar et rangés dans une section intitulée «Aspects du transsexualisme». Ce titre détonne en ce qu'il n'y est pas fait explicitement référence aux transitions FtM alors même que la majorité des propos se rapportent spécifiquement à cette thématique - tendance opposée, donc, au mouvement qui prévaudra par la suite. Y est déjà à l'époque clairement soulevée une interrogation quant à la «rareté des travaux consacrés aux transsexuelles » (Croufer-North, 1981, p. 177), sans qu'aucune hypothèse soit proposée malgré ce constat répété. Il semble néanmoins que la plus grande proportion de transsexuels masculins ne soit pas retenue comme un caractère suffisant à rendre compte de ce phénomène, le psychiatre $\mathrm{P}$. H. Klotz estimant d'ailleurs à cet endroit «que cette prédominance du sexe masculin dans le transsexualisme est seulement une apparence, d'origine socio-culturelle.» (1981, p. 189)

C'est en 1983, dans son ouvrage Horsexe, que Catherine Millot consacre un chapitre entier au «transsexualisme féminin»-l'une des très rares références aussi approfondies sur la question dans l'ensemble de la littérature psychanalytique d'orientation lacanienne. Elle y reprend en les prolongeant deux articles parus en 1981 - «Un cas de transsexualisme féminin» et "Transsexualisme féminin et homosexualité»-, dont le premier envisageait à l'époque que «le transsexualisme chez les femmes ne semble pas s'inscrire dans une problématique symétrique» à celle du transsexualisme masculin, dans la mesure où celui-ci "peut, dans beaucoup de cas, relever de ce "pousse-à-la-femme" de la psychose dont parle Lacan dans L'étourdit» 
(Millot, 1981a, p. 167). Outre la perspective différentielle qu'elle soutient ici entre $\mathrm{MtF}$ et FtM, elle insiste dans le même temps sur l'hétérogénéité propre à ces derniers, qui interdirait la construction d'une « entité clinique distincte et définie du transsexualisme féminin» (Millot, 1981a, p. 167). Si cette affirmation semble exclure toute logique prétendant à pouvoir réunir le transsexualisme féminin sous la bannière d'un opérateur unique, équivalent à la manière dont le pousse-à-la-femme joue pour le transsexualisme masculin, elle confirme également, de manière implicite, que le transsexualisme masculin constituerait de son côté une entité clinique.

Cette démarche différentielle se fait d'ailleurs le point de départ de son ouvrage de 1983, le chapitre consacré aux dites transsexuelles s'ouvrant sur les traits particularisant les FtM au regard des MtF. Difficile ici de ne pas soulever à quel point cette logique même répète ce qui n'a pas manqué d'être reproché à Freud dans sa théorisation sur la féminité, à savoir qu'elle ne se défait jamais d'une comparaison constante au développement psychosexuel masculin, institué par là comme socle et modèle inamovibles.

Deux points majeurs de distinction y sont donc soulevés ${ }^{6}$, dont le rapport à la sexualité, «l'investissement libidinal de l'objet sexuel [étant] beaucoup plus fréquent chez elles que chez les hommes transsexuels, qui n'ont que peu ou pas de désir» (Millot, 1983, p. 104). Cette constatation, reprise par Joël Dor en 1987 et par Maleval presque au mot près en 2014, est également corroborée dans un autre champ par Pat Califia qui tient à souligner le souci constant pour la sexualité perceptible dans les autobiographies de FtM, d'autant plus prégnant que cette dimension serait radicalement absente des témoignages rédigés par les MtF. «Dans les deux premières autobiographies $\mathrm{MtF}$, le fait d'être une femme est lié à une définition limitée et plutôt stricte de la féminité. Du moment qu'une personne ressemble à une jolie femme, semblent dire Jorgensen et Morris ${ }^{7}$, elle est une femme.» (Califia, p. 74). La primauté de ce pôle purement narcissique est souvent mise en exergue dans le discours rapporté des MtF et s'opposerait alors au maintien de l'investissement objectal des FtM, sans que ce point fasse d'ailleurs débat ou tentative d'interprétation. Catherine Millot y voit cependant la raison pour laquelle le transsexualisme féminin a longtemps été perçu comme un déni de l'homosexualité et s'emploiera à différencier les logiques différentes qui soutiennent l'une et l'autre de ces positions selon la distinction nécessaire, ou au contraire la confusion absolue entre pénis et phallus dans le rapport à la partenaire. Pat Califia articule de son côté cette différence entre MtF et FtM aux représentations de genre en vigueur, la confirmation de la virilité 
s'accordant à son exercice en acte et sa possible expression publique, là où la féminité se laisserait réduire à une belle image vouée à être reconnue par l'autre, toute dimension érotique restant voilée par la pudeur qui serait de mise quand il s'agit de sexualité féminine. Censure des éditeurs, autocensure, ou absence effective de tout désir à vocation génitale, aucune hypothèse à ce sujet ne saurait trouver de pertinence tant que n'est dégagé au préalable un problème épineux et plus général qui tend à être passé sous silence: l'écart irréductible qui existe entre les différents dispositifs dans lesquels les propos des personnes concernées se sont déployés, l'absolue hétérogénéité des cadres de parole à partir desquels les différents chercheurs théorisent - à partir d'autobiographies pour Pat Califia, de témoignages ou interviews pour Guillot et Beaubatie, d'entretiens libres pour Catherine Millot - qui ne manque évidemment pas de relever les limites de ce procédé; de patients reçus en psychiatrie générale (Czermak, Frignet) ou au sein de protocoles spécifiques de réassignation sexuelle, de patients en cure psychanalytique (Morel, Godefroy) - comme si ce cadre ne déterminait pas déjà ce qui peut ou doit se dire, ce qui peut ou doit se taire, ce qui peut s'entendre ou non au-delà de ce qui est dit.

Cet aspect différentiel quant à l'investissement narcissique ou objectal n'est jamais que secondaire au regard du trait d'opposition majeur d'avec le transsexualisme masculin sur lequel s'ouvre le chapitre de Millot consacré au transsexualisme féminin: l'aspiration du premier à incarner La femme - soit une position d'exception - y est confrontée au statut d'homme ordinaire qu'appelleraient les transsexuelles de leurs vœux: "être homme, en somme, c'est faire partie du lot commun» (1983, p. 101). Maleval maintiendra cette proposition en $2014^{8}$, formulant que «le transsexuel féminisé s'oriente plutôt vers une position d'exception, celle de La femme toute; tandis que la transsexualisation masculinisante tend à être socialement muette, conforme à la position de celui qui cherche à inscrire sa jouissance dans la loi de l'ensemble qui s'impose aux hommes». Ces propos - qui trouvent leur ancrage théorique dans les formules de la sexuation de Lacan (Lacan, 1972-73) - donnent ici un éclairage tout à fait autre au conformisme forcé et à l'invisibilité dénoncés par Guillot et Beaubatie et mettent ici à découvert une asymétrie radicale entre $\mathrm{MtF}$ et FtM qui n’est étrangement pas soulevée comme telle par les auteurs. Car La femme à laquelle aspirerait le MtF réfute précisément ce qui fait la définition même de la position féminine de Lacan, selon laquelle ta femme, d'être un lieu sans la garantie d'aucun signifiant propre, n'existe pas et ne s'écrit alors qu'à la condition de barrer le La; là 
où le vœu d'être un homme comme les autres chez les FtM confirmerait parfaitement, au contraire, leur inscription dans la position masculine telle que la circonscrit Lacan - soit l'inscription dans un ensemble, un «tous les hommes» marqués de manière commune par la castration de telle sorte que leur jouissance est dite toute phallique. Cette remarque n'a rien d'anodin dans la mesure où c'est bien le rapport à l'exception et à la manière dont la jouissance s'inscrit dans la fonction phallique qui va permettre non seulement la distinction entre les jouissances dites masculine et féminine - en tout-phallique pour la jouissance dite masculine donc, en pas-tout phallique pour la position dite féminine - mais également le repérage de la structure, la jouissance dans la psychose se spécifiant d'être hors castration.

Aussi, organiser le différentiel MtF/FtM autour de l'idée selon laquelle "les uns veulent être $L a$ femme, les autres être un homme» (Millot, 1983, p. 135) inclinerait donc à situer de fait le transsexualisme masculin hors des formules de la position féminine aussi bien que de la position masculine, l'aspiration à incarner La femme faisant alors irrémédiablement résonner le pousse-à-la-femme caractéristique de la jouissance non régulée par le phallus de la psychose.

\section{Schreber: une référence inéluctable et ses conséquences sur le différentiel MtF/FtM}

En effet, à l'exception d'Albert Nguyên qui opère une distinction fort intéressante entre le pousse-à-la-femme et l'enjeu d'une "croyance indéboulonnable en La Femme, problématique, mais qui après tout est le lot de bien des névrosés à l'entrée en analyse » (Nguyên, 2008, p. 177), c'est bien le pousse-à-la-femme qui semble nouer ensemble, par le biais de la référence à Schreber, transsexualisme et psychose.

De par sa constance dans les textes lacaniens portant sur le transsexualisme, cette référence à Schreber tend étrangement à l'instituer comme le MtF le plus célèbre chez les psychanalystes, bien avant Christine Jorgensen ou encore Coccinelle. Cette référence à Schreber a d'ailleurs ceci de paradoxal qu'elle semble inéluctable, exigible, en dépit de la place qu'elle occupe dans l'argumentation - de la simple allusion jusqu'au développement approfondi -, mais surtout de sa fonction - allant de la claire distinction du délire transsexualiste de Schreber d'avec la position transsexuelle (voir Millot, 1983, p. 39 et Rengifo, 2009, p. 207, entre autres références) jusqu'à la constitution de ce même délire comme paradigme du transsexualisme (voir Czermak, 2012, entre autres également). 
Cette dernière pente - qui conçoit la flamboyance du délire de Schreber comme présentant à ciel ouvert des coordonnées structurales communes à la position transsexuelle - est au demeurant celle qui n'accorde aucune place au différentiel Mtf/FtM, dans un effacement qui se présente selon deux versants: soit dans l'absence radicale de toute mention des transitions FtM, le transsexualisme étant uniquement déployé dans le sens MtF sans plus de justification - ce mutisme rendant là les FtM non seulement invisibles, mais comme inexistants. Soit en une simple évocation des FtM en marge du développement théorique principal: à son chapitre sur le transsexualisme, Czermak concède en effet une sous-partie intitulée «Les transsexuelles femmes» (Czermak, 2012, p. 54), s'étendant en tout et pour tout sur une demi-page; quant à Frignet, c'est bien en dehors du corps du texte, en note de bas de page, que se retrouve une remarque sur «la masculinité revendiquée par une femme» (Frignet, 2000, p. 134).

Si la place "en marge» des FtM est à saisir aussi bien au sens strict - comme le rend manifeste cette disposition topologique - qu'au sens figuré, c'est dans la mesure où la concession à y faire référence dans ces cas ne se soutient d'aucune perspective différentielle, mais, tout au contraire, d'une mise en continuité, d'une mise en symétrie entre MtF et FtM, rendant l'étude de ces derniers peu pertinente. C'est bien la communauté de problématique qui est mise en avant, à partir de l'hypothèse selon laquelle le «"hors-jeu" du Phallus interdit aux sujets transsexuels, hommes ou femmes, l'accession à la réalité d'une identité sexuelle ou sexuée établie» (Frignet, 2000, p. 134), ou l'accent porté sur l'" homologie» des transitions FtM avec celle du «transsexuel homme lorsqu'il affirme être "La Vraie Femme"» (Czermak, 2012, p. 54), sans que de plus amples développements ne paraissent nécessaires. Cependant, ce que ce parallélisme laisse non seulement dans l'ombre, mais inexpliqué, c'est bien à quel point la configuration $\mathrm{MtF}$ fonctionne implicitement comme modèle établi quand est pourtant visée l'exploration de «la structure $d u$ transsexualisme en général» (Frignet, p. 133, nous soulignons). Reste ainsi ininterrogé ce qui, dans les transitions FtM, ferait conceptuellement équivalence au «pousse-à-la-femme» à l'œuvre dans les transitions MtF.

\section{L'envie du pénis chez la femme: une controverse houleuse - jusqu'à sa confirmation inédite?}

Si l'expression "pousse-à-l'homme» a bien été tentée, quoiqu'à de rares reprises (Evzonas, 20199; Iddan, 2017; Miller, 2008; Morel, 2000, p. 179), elle n’a néanmoins jamais été élevée au rang de concept, et ne trouve d'ailleurs 
pas de consensus quant à son usage. En effet, Miller la risque à l'occasion d'une interview pour désigner un certain effet impulsé par la modification des stéréotypes socioculturels sur les femmes en général; Claudia Iddan en use pour qualifier le rapport particulier qu'entretient un de ses patients (cisgenre) aux femmes, l'expression se faisant alors l'équivalent d'un "pousseaux-femmes» au cour de sa pratique du polyamour; les deux autres occurrences visent, quant à elles, à qualifier des transitions FtM. Que cette expression soit construite sur le modèle du «pousse-à-la-femme» en une simple déclinaison en symétrie est évident, au point que semble s'abraser du même coup toute nécessité d'avoir à justifier cette même symétrie, ou d'avoir à rendre compte théoriquement du statut de «l'homme» ainsi visé chez les FtM.

Quoique la tentative du "pousse-à-l'homme» apparaisse bien moins explicative que seulement descriptive ou strictement dénominative, elle pourrait témoigner de la nécessité de produire un concept propre aux transitions FtM, équivalent au pousse-à-la-femme pour les MtF. La rareté de cette entreprise et sa timidité théorique semblent cependant indiquer tout au contraire la non-pertinence d'un tel néologisme conceptuel, quand un concept déjà inscrit dans la théorie psychanalytique depuis Freud apparaît comme prêt à emploi pour rendre compte de ce qui pousserait une femme à être un homme: l'envie du pénis.

«Il est vrai que la femme rêve d'être un homme» (Godefroy, 2014, p. 109); c'est par cette assertion donnée comme une évidence qu'Hélène Godefroy introduit en effet l'élaboration théorique qu'elle propose au sujet des «femmes transgenres». L'effarement est sans mesure face à l'emploi d'une telle universelle hissée au rang de vérité, en ce que la rapidité de la formule et les propos qui suivent tendent à escamoter les controverses qui agitèrent le mouvement psychanalytique au sujet des hypothèses de Freud selon lesquelles la masculinité serait primaire chez la petite fille et l'envie du pénis la cicatrice inévitable de sa castration en même temps que le pivot de son développement psychosexuel. Que la petite fille soit un petit homme (Freud, 1932, p. 201) jusqu'à la découverte de sa castration, que la féminité n'apparaisse jamais autrement que comme un horizon sans garantie, sans cesse menacé de régression à cette masculinité originaire, que l'envie du pénis constitue pour la femme un roc indépassable; tels sont les fondements de la construction théorique de Freud à l'origine des contestations. Les débats houleux qui sévirent dans les années 20 et 30 entre Vienne et Londres - dont Lacan a résumé l'enjeu principal par la question de savoir si la femme était 
«born or made ${ }^{10} »(1966$, p. 729$)$ - ont d'ailleurs ceci de particulier que jamais l'envie du pénis ne fut remise en cause en tant que telle, mais exclusivement dans sa fonction: primaire selon Freud, secondaire selon le mouvement anglo-saxon. Cette contestation des théories freudiennes, qui ne saurait se réduire à une voix homogène, s'accorde toutefois sur la perspective d'envisager le complexe de masculinité et le phallicisme de la petite fille comme des formations secondaires, comme des solutions défensives au regard d'une sexualité féminine primaire engendrant des angoisses telles que la masculinité s'offrirait alors comme une véritable issue permettant à la petite fille de fuir hors de sa féminité initiale ${ }^{11}$. L'importance de l'envie du pénis se trouve certes maintenue dans cette perspective, mais au prix d'un complet renversement de ses enjeux. Cette bascule n'a cependant rien de commun avec l'ironie mordante de Luce Irigaray, dans les années 70, lorsqu'elle s'attelle à déconstruire avec virulence les propositions freudiennes sur la féminité (Irigaray, 1974), mettant à découvert de façon systématique à quel point le carcan phallique qui enserre la sexualité féminine ne serait jamais imputable qu'aux représentations masculines à partir desquelles elle est conçue - là même où elle y échapperait, résolument.

Sans déployer plus avant ces critiques largement connues, leur évocation a pour double fonction de rappeler d'une part que les fondements de la construction théorique de Freud qui traite la féminité à partir de la masculinité n'ont cessé de faire scandale et de susciter les critiques à l'intérieur de la psychanalyse aussi bien qu'à l'extérieur de son champ; d'insister, d'autre part, sur le fait que lesdites critiques, de par leur recours à l'idée d'une «nature» féminine, ou de par leur appui sur une anatomie qui dessinerait en tant que telle une spécificité, sont aujourd'hui sévèrement mises elles-mêmes à la critique, pour ce que la proposition d'une féminité épurée de fait de masculinité et fondamentalement distincte de toute organisation phallico-masculine révèle d'une conception naturaliste ou essentialiste du sexe. Les transidentités et le mouvement queer ont en effet entraîné avec eux une nouvelle perspective critique à l'égard des conceptions de la différence des sexes, en mettant en exergue le fait qu'elles sont toujours historiquement situées, sans manquer de mettre à découvert leur dimension irréductible de construction. Si les théories sur le genre subvertissent les représentations communément admises sur la différence des sexes, elles ne sont pas sans convoquer également les théories psychanalytiques pour en dénoncer les limites prétendument normatives - selon une conception de la norme qui ne se distinguerait pas de la norme sociale. 
C'est à cet endroit qu'un abord différentiel des transitions trouve précisément tout son intérêt, en ce que ces dernières mettent à l'épreuve de façon tout à fait distincte les théories psychanalytiques sur la masculinité et la féminité; car si les MtF font figure d'impossible au regard du développement psychosexuel masculin normé par l'Eedipe tel que le conçoit Freud, les FtM trouvent alors à se spécifier de ne heurter en rien les propositions freudiennes sur la féminité - jusqu'au point même de venir illustrer de façon tout à fait inédite certains propos de Freud sur le complexe de masculinité, les cas de figure qu'ils désignent demeurant autrement difficiles à saisir. C'est ainsi que la situation que décrit Freud - «la petite fille refuse d'accepter le fait de sa castration, elle s'entête dans sa conviction qu'elle possède bien un pénis et est contrainte par la suite à se comporter comme si elle était un homme» (Freud, 1925a, p. 127) - pourrait, selon Nicolle KressRosen, "aujourd'hui s'appliquer au transsexualisme féminin de manière assez précise» (Kress-Rosen, 1999, p. 218). Sylvie Sesé-Léger repère pour sa part «le cas de la demande transsexuelle» (Sesé-Léger, 1996, p. 480-81) dans la citation qui précède: «l'espoir d'obtenir un jour, malgré tout, un pénis et ainsi de devenir semblable ${ }^{12}$ aux hommes peut se maintenir jusqu'à une époque incroyablement tardive et devenir le motif d'actes étranges qui sans cela seraient incompréhensibles» (Freud, 1925a, p. 127).

Ce que ne relève pas Hélène Godefroy dans son hypothèse de théorisation concerne bien la confirmation inattendue que les FtM apportent aux théories contestées de Freud sur la féminité, à partir d'une expression sans fard de l'envie du pénis qui n'aurait connu aucune équation symbolique et qui ne nécessiterait alors aucun déchiffrement. Ce traitement particulier de l'envie du pénis, comme à ciel ouvert - s'il est possible d'user de cette expression malgré le risque qu'elle entraîne de toujours implicitement évoquer la psychose - serait certes à entendre, dans une perspective freudienne, comme non-résolution du complexe de masculinité, mais encore et surtout comme son illustration la plus épurée.

Freud n'a pas manqué lui-même de lier, quoique hâtivement, la psychose avec cette configuration particulière du complexe de masculinité ${ }^{13}$, rendant difficile l'appréhension de cette dernière comme strict avatar de l'envie du pénis, tant les dimensions de Neid (envie) ou de Wunsch (désir) ${ }^{14}$ du pénis disparaissent ici, écrasées par la conviction d'un avoir qui n'est mis ni au passé, ni au futur. L'équivalence proposée par Kress-Rosen entre la citation qu'elle convoque chez Freud et la situation FtM est à ce titre à lire avec précaution, dans la mesure où la «conviction» de «bien posséder un 
pénis» ne caractérise certainement pas la totalité desdites «transsexuelles». De son côté, la citation convoquée par Sesé-Léger est abusive, les transitions FtM ne subsumant évidemment pas l'ensemble des déclinaisons possibles du complexe de masculinité; le cas Dora, ou encore celui de la jeune homosexuelle, pour n'évoquer qu'eux, sont là pour témoigner de ce que l'envie du pénis et les identifications masculines classiquement en jeu n'entravent en rien le fait de se reconnaître et de se dire femme.

\section{FtM et psychanalyse: hypothèses sur un silence théorique}

Il n'en demeure pas moins que si les transitions FtM ne semblent pas constituer une énigme pour la psychanalyse, au regard de la rareté des études spécifiques qui leur sont consacrées, c'est bien dans la mesure où elles évoquent immédiatement le complexe de masculinité et l'envie du pénis inscrits dans le développement psychosexuel féminin, où elles apparaissent donc en conformité avec les propositions freudiennes sur la féminité; une conformité telle que Kress-Rosen ira d'ailleurs jusqu'à opposer la "banalité» des tableaux présentés par les transsexuelles à l'aspect «contre-nature, du point de vue névrotique», des transsexuels (1999, p. 209). Il conviendrait de préciser que ce tableau ne doit sa «banalité» qu'à l'homologie qu'il présente avec les descriptions freudiennes, côté femme - au point alors que SeséLéger se contente de juxtaposer un certain nombre de citations de Freud sur près d'une page (1996, p. 481), sans prendre la peine de les commenter, tant celles-ci parleraient déjà, de façon quasi transparente, de son patient FtM Dominique - et côté homme, un aspect qualifié de "contre-nature» d'une façon fort peu heureuse, en ce que la «nature» en question ne se réfère à rien d'autre qu'à la théorie freudienne concernant le développement psychosexuel masculin et sa résolution névrotique.

Il convient à cet endroit de préciser à nouveau le cadre du questionnement qui est ici au travail: il s'agit non pas de discuter de la pertinence des hypothèses qui ont été posées pour rendre compte des enjeux propres aux transitions FtM - et il s'avèrera que la lecture de ces transitions à partir de l'envie du pénis est loin de faire consensus -, mais bien de mettre à découvert les automatismes théoriques issus de la théorie psychanalytique de la différence des sexes, en tant qu'ils ordonnent à notre insu une appréhension différentielle du transsexualisme. Le raccourci en jeu pourrait se formuler de la sorte: là où le transsexualisme $\mathrm{MtF}$ convoque spontanément la référence à Schreber, les transitions FtM font quant à elles écho au développement psychosexuel féminin dans ce qu'il revêt de plus traditionnel, comme 
avatar particulier du complexe de masculinité. Cette proposition ouvre à deux lignes de remarques, l'une qui concerne la psychosexualité féminine et sa place tout à fait singulière dans la théorie psychanalytique, et l'autre qui, non sans lien, concerne le diagnostic différentiel.

Que le transsexualisme chez la femme ne produise manifestement pas le même embarras et, partant, la même vivacité d'intérêt que le transsexualisme masculin peut trouver à s'éclairer à partir de l'affirmation de Freud selon laquelle «la bisexualité, dont nous affirmons qu'elle est propre à la prédisposition humaine, ressort beaucoup plus nettement chez la femme que chez l'homme» (Freud, 1931, p. 12). Une bisexualité qui opère moins en synchronie qu'en une véritable succession pour la petite fille à partir de la découverte de la différence des sexes, en une bascule qui se traduira par un changement d'identité (le "petit homme » se muant, ou non, en femme) associé à un changement de sexe-le clitoris, conçu comme organe masculin, devant céder son érogénéité au vagin, seul organe "proprement féminin» (Freud, 1931, p. 12). Le développement psychosexuel féminin n'en devient-il pas alors étrangement comparable à un parcours de réassignation sexuelle, à une trajectoire trans' - MtF, pour le coup? La notion de choix d'objet est également délicate à saisir, en ce que la découverte de l'importance primordiale de la relation à la mère pour la petite fille ne saurait pour autant se laisser décrire comme une relation homosexuelle féminine, dans la mesure où elle relie une petite fille phallique à une mère phallique; dire de cette relation qu'elle relèverait de l'homosexualité masculine ne rendrait pas la description plus pertinente, car s'il est largement admis que le phallus n'est pas le pénis, encore s'agirait-il de souligner que le phallique n'est pas synonyme de masculin.

Ainsi, l'abord de la féminité ne conduit-il pas seulement à troubler ce que serait l'identité féminine - fondamentalement masculine, selon Freud mais encore à subvertir la notion d'homosexualité - tout du moins dans sa définition commune. En effet, les critères en jeu dans les qualifications d'hétéro- ou d'homosexualité prennent communément en considération «le genre des deux partenaires et définissent leur relation en termes d'identité/altérité» (Hérault, 2010, p. 288), critères alors mis en aberration par les transidentités dans la mesure où cette logique supposerait qu'avec la transition identitaire s'opère nécessairement une transition de dénomination de l'orientation sexuelle, là même où le genre de l'objet désiré n'aurait quant à lui pas changé. L'«homosexualité» de la relation mère-fille ne présage quant à elle en rien du genre ni de la mère ni de la fille, si ce n'est que ce sont les mêmes ${ }^{15}$. 
C'est donc une véritable fragilisation des repères de l'«identité» et de la sexualité qu'imprime l'étude de la féminité, au point que l'hypothèse du complexe d'CEdipe comme noyau de la névrose est elle-même remise en cause par Freud à la fin de son élaboration en ce qui concerne spécifiquement les trajectoires féminines - ce qui démontre à quel point la féminité est dès l'origine le lieu privilégié d'ébranlement des certitudes théoriques pour la psychanalyse. Si Gayle Rubin n'a pas hésité à considérer la psychanalyse comme une théorie du genre (1975, p. 68), il est possible de supposer que c'est certainement l'étude de la féminité qui, une fois tombée l'illusion de tout parallélisme avec la masculinité, a spécifiquement entraîné Freud à «queeriser» sa théorie, faisant alors de la féminité, si ce n'est un lieu trans', du moins un lieu qui ne lui est pas tout à fait étranger.

Le point le plus regrettable de la théorie freudienne sur la féminité, cela dit dans une perspective lacanienne, est d'avoir fabriqué du «toutes les femmes» à partir du pivot unique qu'est l'envie du pénis - auquel Lacan substituera, au contraire, le rapport contingent de chaque femme à la castration. Les enjeux phalliques chez la femme ne sont pour autant pas évacués, bien qu'ils soient dédoublés dans le registre de l'être et de l'avoir, une fois radicalisée la distinction entre pénis et phallus, encore confondus chez Freud. Ce dernier champ de l'avoir phallique ne reste d'ailleurs pas réservé aux hommes, ni réductible pour les femmes à la seule solution de la maternité, comme Lacan semble le suggérer lorsqu'il formule qu'il «reste à prendre de la graine du naturel avec lequel telles femmes se réclament de leur qualité d'hommes, pour l'opposer au style de délire du transsexualiste masculin» (Lacan, 1966, p. 735). Cette citation qui pourrait être une référence au transsexualisme féminin - si ce n'est qu'elle se situe explicitement dans une partie consacrée à l'homosexualité féminine ${ }^{16}$ - propose ainsi un trait différentiel qui oppose le «délire» $\mathrm{MtF}$ au «naturel» FtM, ce qui n’est pas sans confirmer le statut particulier qu'occupe la position féminine dans la théorie psychanalytique, à être ainsi conçue comme fondamentalement hybride. Est à ce sujet souvent souligné que la particularité du transsexualisme féminin réside dans cette «réduction du phallus au pénis» (Millot, 1983 , p. 122); outre que cette position se distinguerait par ce biais de l'homosexualité féminine telle que Freud et Lacan en ont proposé la lecture, ce point met surtout en exergue qu'il n'est nul besoin de «l'avoir» anatomiquement pour que la confusion pénis-phallus soit consommée pour un sujet. Cet élément supposerait par ailleurs un traitement différent de «l'erreur commune»-dont Lacan dit que le «transsexualiste» veut se libérer (Lacan, 
1971-72, p. 17); car si c'est moins de l'organe que du signifiant dont le MtF ne veut plus, qu'en est-il pour les FtM?

\section{Traitement différentiel des transitions ou hypothèse unitaire: la question diagnostique}

Pour en revenir à l'apparente intimité entre l'envie du pénis et les enjeux FtM, force est de reconnaître que cet axe de lecture ne fait pas consensus: "On ne peut valablement considérer que [la] spécificité [du transsexualisme féminin] viendrait se fondre dans la revendication féminine commune, celle du Penisneid» (Jean, 1997, p. 29). Geneviève Morel formulera très clairement son doute à l'écoute de son patient Ven racontant le souvenir-écran qui semble avoir cristallisé sa transition, la vision d'un petit garçon urinant debout: «il avait alors pensé que c'était ce qu'il voulait être: un garçon» (Morel, 2000, p. 201). En dépit de l'écho spontané provoqué par cette scène avec les propos de Freud de 1925, «à la vue du pénis d'un frère ou d'un camarade de jeux, dit Freud, "elle a jugé et décidé. Elle a vu cela, sait qu'elle ne l'a pas et veut l'avoir”» (Morel, 2000, p. 201), s'ouvre alors l'alternative de repérage suivante: complexe de castration féminin ou forclusion de la signification phallique? Si la réponse à cette question ne fait pas mystère, dans la mesure où la description de ce travail analytique prend place dans un chapitre intitulé «Sexuation et psychose», c'est bien la question du diagnostic différentiel qui est ici en jeu, entre névrose et psychose.

C'est pourtant une troisième voie qui semble explicitement privilégiée par les tenants d'un différentiel entre MtF et FtM, celle de la perversion. C'est tout du moins l'hypothèse que semble soutenir Joël Dor (1987, p. 256), quand bien même cela le mettrait aux prises avec ce qui lui apparaît comme une aberration théorique - la perversion, dans son abord structural de déni de la castration, pouvant difficilement s'appliquer aux femmes «en raison du rapport que la femme entretient nécessairement au réel de l'absence phallique» (Dor, 1987, p. 265). Kress-Rosen propose quant à elle l'hypothèse d'une Verleugnung «au féminin» (Kress-Rosen, 1999, p. 217), autrement dit un «fétichisme partiel» à partir d'un déni de la castration qui ne se généraliserait pas à toutes les femmes, mais qui toucherait exclusivement leur propre personne: «elles seraient en fait les seules femmes fétichistes, comme si leur identification forcenée à l'homme allait jusqu'à leur faire adopter leur mode de défense contre l'angoisse de castration» (Kress-Rosen, 1999, p. 219). Le diagnostic de psychose serait quant à lui réservé aux $\mathrm{MtF}$, sans que cela fasse l'objet du moindre doute ou questionnement selon les auteurs en question. 
Ce point est primordial en ce qu'il souligne que le soutien d'une perspective différentielle $\mathrm{MtF} / \mathrm{FtM}$ s'assortit immanquablement d'un différentiel quant au diagnostic de structure, voire qu'il en tire proprement sa pertinence. A contrario, l'équivalence posée par la majorité des auteurs entre transsexualisme et psychose, dans la mesure où la position transsexuelle se spécifierait d'être «hors sexe», efface de fait tout l'intérêt d'un abord différencié des transitions.

Insiste néanmoins l'hypothèse fantomatique et timide d'un autre diagnostic, qui n'est jamais formulé autrement qu'en termes d'interrogation. Transgressant ces lignes de partage apparemment très claires, cette hypothèse peut être énoncée par des auteurs soutenant une conception du transsexualisme unique aussi bien que par des auteurs différentialistes; ni psychose, ni perversion, c'est bien l'hystérie qui insiste de cette manière, et qui trouve un relief tout à fait particulier de n'être jamais évoquée qu'en rapport aux transitions FtM.

Si Erik Porge (2000) pointe, tout en la réfutant, cette tendance à réserver la psychose côté MtF et à envisager l'hystérie côté FtM, si Geneviève Morel en vient quant à elle à discuter cliniquement de cette question par rapport à son patient FtM Ven - «J'ai hésité sur le diagnostic de structure [...] Je doutais [...] J'ai donc pris le temps d'être sûre qu'il ne s'agissait pas d'une hystérie, mais d'une psychose.» (2000, p. 204) - certes pour la réfuter également, ce qui importe cependant est le fait que la question de la névrose émerge exclusivement quand il est question de transition dans ce sens. En effet, la seule alternative possible au diagnostic de psychose pour le transsexualisme dit «masculin» est celui de perversion ${ }^{17}$ - mais cette alternative relève plutôt d'une distinction radicale entre transsexualisme et transvestisme (tel le patient de Rengifo), qui recouvre une autre discussion, celle entre un transsexualisme "authentique», un transsexualisme "vrai», ou primaire, et un transsexualisme secondaire.

Quand elle n'est pas clairement infirmée, l'hypothèse de l'hystérie conserve cependant un statut trouble, à la fois très présent, toujours précautionneux et parfois obscur. Kress-Rosen n'hésitera pas à annoncer en introduction à la partie de son ouvrage dévolue au transsexualisme qu'elle s'attachera à «dissocier les formes masculine et féminine du transsexualisme» et que «l'on ne s'étonnera pas de retrouver, du côté féminin, la problématique hystérique» (1999, p. 186), alors même que le chapitre en question ne suit pas cette veine, mais bien celle d'un type de perversion spécifiquement féminin. «Alors hystérie ou perversion?» (Sesé-Léger, 1996, p. 475); 
cette question se pose selon l'auteure à chaque rencontre avec un FtM - et si elle n'y répond pas explicitement, l'hystérie semble avoir sa faveur pour son patient Dominique. Mais la position la plus intrigante est sans conteste celle de Catherine Millot (1983). Alors même que l'architecture de son ouvrage se fonde sur une distinction entre les transitions selon leur orientation, que cette distinction est étoffée par les différences de fonctionnement qu'elle repère, que la question de l'hystérie fait son entrée, de manière insistante, dans sa partie sur le transsexualisme féminin - là où elle n'est à aucun moment évoquée concernant les $\mathrm{MtF}$-, c'est la logique même de cette construction qu'elle va finir par remettre en cause explicitement en conclusion. Cette remise en cause s'opère au profit d'une hypothèse unitaire et commune aux FtM et aux MtF, qui rompt non seulement avec le différentiel d'abord supposé, mais également avec le constat, maintes fois répété, de l'absolue diversité des cas, particulièrement manifeste chez les FtM.

Il m'avait semblé pouvoir faire l'hypothèse d'une radicale différence de position des femmes transsexuelles par rapport au transsexualisme masculin. Les uns veulent être $\mathrm{La}$ femme, les autres être un homme. La dissymétrie semblait évidente. Or, après avoir entendu les femmes transsexuelles, en particulier Gabriel, je serais plutôt portée à souligner la parenté de la position des transsexuels des deux sexes, en ce qui concerne le rapport au phallus et l'identification à ce qu'il incarne d'hors-sexe, voire d'hors-corps (Millot, 1983, p. 135).

Telle est l'hypothèse uniforme sur laquelle elle conclut à partir de la bascule produite par les propos de Gabriel, seul FtM avec lequel elle s'entretient qui prendra contact avec elle de lui-même, dans le but déclaré de lui révéler la «vérité sur le transsexualisme» (Millot, 1983, p. 127). Outre le fait que le discours de Gabriel a ceci de très moderne qu'il rejoint le discours actuel sur la remise en cause de la binarité ( «la vérité sur le transsexualisme, c'est que contrairement à ce qu'ils prétendent en général - avoir une âme d'homme prisonnière d'un corps de femme [ou l'inverse] - les transsexuels ne sont ni hommes ni femmes, ils sont différents»), il est tout à fait frappant que Catherine Millot l'ait pris au pied de la lettre, c'est-à-dire comme ayant valeur de vérité pour tout transsexuel.

L'hypothèse unique qu'elle formule alors, et qui donnera le titre de son ouvrage, Horsexe, a ceci d'ambigu qu'elle semble refouler plutôt que neutraliser ses hypothèses précédentes. D'une part, elle est en effet démentie par 
un retour immédiat à une perspective différentielle, qui conduit de nouveau Millot à parler distinctement des hommes transsexuels et des femmes transsexuelles (Millot, 1983, p. 136) et, d'autre part, «l'épure» que constituerait la vérité du transsexualisme selon Gabriel en vient à recouvrir l'hypothèse de l'hystérie, pourtant mentionnée dans l'introduction générale de son étude du transsexualisme féminin et en rapport à la position particulière de Victor - autrement dit à la faire disparaître sans être l'objet de la moindre discussion. Tout se passe comme si l'hétérogénéité des enjeux pouvant conduire à une transition devait être réduite à une formulation théorique unique, comme si la possibilité de ressorts multiples n'était pas envisageable, dans une logique qui par contrecoup produit le transsexualisme comme entité unique et homogène et exclut le cas par cas.

\section{Conclusion}

La littérature analytique sur la question trans' oppose un intérêt massif suscité par les MtF à la rareté des travaux ou évocations des FtM, fracture qui a été rapportée à la manière dont ces transitions font résonner différemment les théories psychanalytiques sur la féminité et sur la masculinité. Par l'écho inédit produit avec la théorie freudienne sur la féminité, les transitions FtM ne semblent pas constituer un point d'énigme en psychanalyse tant la masculinité chez la femme n'est en rien étrangère à son développement - tandis que la féminisation chez l'homme convoque immédiatement comme référence la position d'exception de Schreber.

Il est intéressant de constater à ce sujet que le mouvement transgenre infléchit considérablement les critiques qui étaient jusque-là opposées aux théories psychanalytiques sur la féminité, notamment la réaction contre le postulat freudien d'une masculinité primaire chez la femme. Chuterait, avec ce renversement de perspective, l'accusation majeure qui était faite à Freud, celle de n'avoir su saisir la féminité indépendamment d'une lecture phallocentrée, soupçonnée alors de se confondre avec une lecture misogyne. De nouvelles perspectives de lectures pourraient tout du moins être faites quant aux propositions freudiennes à partir de la remise en cause impulsée par ces critiques - à condition de considérer que ces critiques, au même titre que les théories sur la différence des sexes, sont elles-mêmes historiquement situées, et que leurs conditions d'émergence sont également produites par un certain cadre politique, social et culturel.

Toutefois, l'éclipse subie par les FtM au profit d'une focalisation presque exclusive sur les MtF se soutient plutôt théoriquement d'une conception 
unitaire du transsexualisme - qui retire de fait toute pertinence à une approche différentielle des transitions, sans que ne s'éclaire pour autant la raison pour laquelle les $\mathrm{MtF}$ en constituent le seul modèle. L'étude de la valeur accordée ou non au différentiel FtM/MtF selon les auteurs a par ailleurs conduit à mettre en exergue que les abords distincts du transsexualisme sont indissociables de considérations diagnostiques: le déchiffrage des transitions du côté d'une position hors-sexe se réfère en effet systématiquement à la structure psychotique, selon les quelques indications données par Lacan sur le transsexualisme, tandis que l'étude des FtM dans une perspective différentielle voit se multiplier les hypothèses diagnostiques, entre névrose, psychose et perversion.

S'il ne semble pas anodin que les FtM soient le lieu électif de cette indécision, de cette suspension des certitudes diagnostiques, il paraîtrait plus que pertinent que ce mouvement d'hésitation diagnostique s'élargisse aux transitions en général, une fois admis que l'étude des transidentités en tant que phénomène ne saurait se résoudre par un diagnostic de structure unique - une fois admis, donc, que l'hétérogénéité des transitions n'est jamais que fictivement unifiée par la formule traditionnelle mettant en jeu la conviction d'appartenir à l'autre genre. C'est bien le transsexualisme en tant que structure clinique qui est ici remis en cause par la multiplicité des voix trans' et des solutions singulières - ce que Castel énonce très clairement par la formule suivant laquelle " "Le" transsexualisme, ça n'existe pas», en tant qu'il n'existe pas «de classe homogène où tombent tous les vœux de "changer de sexe” »(Castel, 2015, p. 64). Une fois écarté l'écueil qui existe à faire consister «le» transsexualisme comme une identité, la perspective qui s'ouvre engage à «interroger, voire réviser les certitudes cliniques qui avaient cours jusquelà en matière de transsexualisme» (Nguyên, 2008, p. 171). Reste donc à prendre acte, théoriquement, qu'à l'instar de toute clinique, les phénomènes trans' relèvent du cas par cas, au risque sinon de raccourcis diagnostics - au risque, donc, de dénaturer par là même ce qui fonde les spécificités du diagnostic de structure en psychanalyse, dans ses coordonnées et sa fonction particulières dans le travail analytique et le maniement du transfert ${ }^{18}$.

Fanny Chevalier fanny.chevalier@univ-amu.fr 


\section{Notes}

1. Si Giami (2011, p. 269) souligne que «désigner les personnes trans par le sexe attribué à la naissance ou par leur identification de genre souhaitée et revendiqueé» constitue «un des marqueurs de positions spécifiques occupées dans le champ» (tout comme le choix des termes «transsexuel», "transgenre», etc.), il est possible d'objecter que la position en question n'est pas toujours si évidente à repérer à partir de ces seuls marqueurs - par exemple quand certains des auteurs qui parlent de «femmes transsexuelles» en psychanalyse usent par ailleurs de pronoms masculins pour les désigner.

2. Du fait - du moins en partie - des dates de publication des textes qui seront convoqués dans ce travail.

3. Ce qui ne règlera pas pour autant le problème de l'exhaustivité. Il est utile de préciser que certains textes cités qui ne font pas explicitement référence à Lacan ont cependant été inclus par voisinage en quelque sorte, dans la mesure où les auteurs de ces textes ont par ailleurs publié dans des revues lacaniennes sur la question dudit transsexualisme.

4. "Le Manuel diagnostique et statistique des troubles mentaux évoque un sex-ratio qui se situerait entre $1 \mathrm{FtM}$ pour 2,5 MtFs et $1 \mathrm{FtM}$ pour $5 \mathrm{MtFs}$. Cette estimation se fonde sur les traitements avec réassignation de sexe, la requête de cette chirurgie par le patient étant considérée comme l'un des symptômes cliniques. Or, les technologies destinées aux hommes trans' sont moins développées et l'opération génitale est, en conséquence, rarement exigée par les tribunaux pour leurs changements d'état civil à la différence de ceux des femmes. Les personnes en transition ne souhaitant pas nécessairement avoir recours à cette chirurgie si elle n'est pas une condition d'obtention de leurs papiers d'identité, le décalage de visibilité médicale entre MtFs et FtMs s'entretient dans un cercle vicieux.» (Beaubatie, 2016 b, p. 138-139).

5. Les termes «transsexualisme féminin», "(femmes) transsexuelles» ou "femmes transgenres» seront employés dans le texte quand ils sont ceux utilisés par les auteurs cités. Sera sinon privilégiée l'expression «FtM».

6. Nous laisserons de côté ici les distinctions d'ordre plus général, concernant notamment les opérations chirurgicales génitales dont la réussite plastique, esthétique et fonctionnelle n'est pas la même selon l'orientation de la transition.

7. Pat Califia fait ici référence à deux autobiographies célèbres de MtF, rédigées par Christine Jorgensen et Jan Morris.

8. Voir également Maleval (2019), en particulier le chapitre intitulé «Du fantasme de changement de sexe au sinthome transsexuel» (p. 186-208).

9. Soulignons que cette dernière référence bibliographique s'est clandestinement glissée dans le corpus choisi, précisément de par son titre - «La violence "pousse-àl'homme" - qui décline explicitement le concept lacanien de "pousse-à-la-femme», alors même que la théorie lacanienne n'est manifestement pas l'orientation de l'auteur.

10. Reformulant par là les propos conclusifs de Jones dans son article «Sexualité féminine primitive»: «en fin de compte, il s'agit de savoir si une femme naît femme ou si elle le devient» (Jones, 1935, p. 452).

11. Une fuite «hors de» la féminité («Flucht aus der Weiblichkeit», selon le titre original) et non «devant» la féminité, comme le propose de façon erronée la traduction française de l'article de Karen Horney (1926).

12. La traduction des CEuvres complètes - «L'espoir de finir par avoir tout de même un jour un pénis et par là de devenir égale à l'homme peut se maintenir jusqu'en des temps invraisemblablement tardifs» (Freud, 1925 b, p. 196) - infléchit quelque peu le sens à accorder à cette phrase. Si la version originale de Freud ne semble pas permettre de 
trancher entre ces deux traductions, le texte de 1931 en revanche est très clair: «l'espoir de recouvrer un jour un pénis se maintient jusqu'en des temps incroyablement tardifs, est érigé en finalité de la vie, et la fantaisie d'être envers et contre tout un homme demeure souvent un élément configurateur» (1931, p. 14).

13. Celle-ci s'entendant comme déni de la castration: «Ou bien intervient le processus que j'aimerais désigner comme déni qui, dans la vie d'âme enfantine, semble n'être ni rare ni très dangereux, mais qui, chez l'adulte, introduirait une psychose. La fille refuse d'admettre le fait de sa castration, se raidit dans la conviction qu'elle possède malgré tout un pénis et est contrainte de se comporter par la suite comme si elle était un homme.» (Freud, 1925 b, p. 196)

14. Terme qui semble privilégié par Freud dans De la sexualité féminine, sans que ce glissement n'apparaisse comme significatif, si l'on en croit l'entrée consacrée à l'«Envie du pénis» dans le Vocabulaire de la psychanalyse.

15. L'alternative proposée consisterait alors à choisir des termes qui restent «muets sur le genre du désirant et focalisent l'attention sur la qualité sexuée du sujet-objet de désir (gynéphilie, androphilie, autogynéphilie, autoandrophilie, etc.)» (Hérault, 2010, p. 288).

16. Comme le souligne également Maleval (2014), Miller n'hésite pas à y lire que Lacan établit par là une "disjonction complète entre le transsexualisme masculin et le transsexualisme féminin. Dans le transsexualisme masculin, il y a du théâtre, tandis que le transsexualisme féminin, qui aspire à l'homme, le fait avec beaucoup de naturel parce que c'est dans la veine même de la sexualité féminine» (Miller, 1982-1983, p. 136).

17. À l'exception notable d'un article de Jean-Jacques Rassial, qui déchiffre la demande de castration et de féminisation formulée par un jeune homme pour lequel il fait l'hypothèse de l'hystérie comme étant l'effet produit par une certaine «mauvaise rencontre». (Rassial, 2004, p. 31). Ce patient reçu en analyse sera néanmoins d'emblée distingué des «quelques transsexuels» rencontrés dans ce cadre par l'auteur, en ce qu'il paraissait «loin de la certitude paranoïaque d'une identité féminine» (Rassial, 2004, p. 29).

18. Cette remarque ouvrirait à la nécessité d'une discussion plus approfondie concernant le diagnostic psychanalytique dans sa rupture d'avec le diagnostic psychiatrique, notamment en ce qui concerne la psychose. Détacher la question diagnostique de la dimension trans' en tant que telle, pour la resituer au «un-par-un» du sujet en analyse, restituerait au diagnostic de structure, quel qu'il soit, sa valeur non déficitaire et non pathologisante. Cette précision permettrait d'objecter aux critiques «d'injure diagnostique» (Ayouch, 2015) ou de «maltraitance théorique» (Sironi, 2011), qui ne sont pas sans produire le risque de générer en contrepartie une censure conceptuelle.

\section{Références}

Alessandrin, A. (2012). Le transsexualisme: une catégorie nosographique obsolète. Santé publique, 24 (3), 263-268. doi: 10.3917/spub.123.0263

Ayouch, T. (2015). Psychanalyse et transidentités: hétérotopies. L'évolution psychiatrique, 80 (2), 303-316.

Beaubatie, E. (2016 a). Trans'. Dans J. Rennes (dir.), Encyclopédie critique du genre: Corps, sexualité, rapports sociaux (p. 640-648). Paris: La Découverte.

Beaubatie, E. (2016 b). Psychiatres normatifs vs. trans' subversifs: Controverse autour des parcours de changement de sexe. Raisons politiques, 62 (2), 131-142. doi: 10,3917/ rai.062.0131

Califia, P. (1997). Le mouvement transgenre. Paris: EPEL. 2003.

Castel, P.-H. (2015). La métamorphose impensable après coup. Penser/rêver, 27, 59-67. 
Croufer-North, E. (1981). Le cas «Sébastien». Ornicar, 22-23, 177-183.

Czermak, M. (1986). Précisions sur la clinique du transsexualisme. Dans Passions de l'objet. Paris: Éditions Joseph Clims.

Czermak, M. (2012). Patronymies: Considérations cliniques sur les psychoses. Toulouse: Erès.

Dor, J. (1987). Structure et perversions. Paris: Denoël.

Evzonas, N. (2019). La violence "pousse-à-l'homme»: approche clinique d'une subjectivation transmasculine. Psychologie clinique et projective, 25 (1), 11-36. doi: 10,3917/ pcp.025.0011

Frignet, H. (2000). Le transsexualisme. Paris: Desclée de Bouwer.

Freud, S. (1925 a). Quelques conséquences psychiques de la différence anatomique entre les sexes. Dans La vie sexuelle (p. 123-132). Paris: Presses universitaires de France, 2005.

Freud, S. (1925 b). Quelques conséquences psychiques de la différence des sexes au niveau anatomique. Dans Euvres complètes françaises de psychanalyse, vol. XVII (p. 190-202). Paris: Presses universitaires de France, 1992.

Freud, S. (1931). De la sexualité féminine. Dans Euvres complètes françaises de psychanalyse, vol. XIX (p. 9-28). Paris: Presses universitaires de France, 2004.

Freud, S. (1932). La féminité. Dans Euvres complètes françaises de psychanalyse, vol. XIX (p. 195-219). Paris: Presses universitaires de France, 2004.

Giami, A. (2011). Identifier et classifier les trans: entre psychiatrie, épidémiologie et associations d'usagers. L'information psychiatrique, 87 (4), 269-277. doi: 10,3917/ inpsy.8704.0269

Godefroy, H. (2014). Les femmes transgenres. Une clinique à la marge. Dans G. Chaboudez (dir.), Actualités de la psychanalyse (p. 107-112). Toulouse: Éditions Érès. doi: 10,391 7/ eres.gilli.2014.01.0107

Guillot, J. et Beaubatie, E. (2012). L’invisibilité FtM: aspects sociaux et politiques. Dans A. Alessandrin (dir.), La Transidentité: des changements individuels au débat de société (p. 71-92). Paris: L'Harmattan.

Hérault, L. (2010). Usages de la sexualité dans la clinique du transsexualisme. L'Autre, 11 (3), 279-291. doi: 10.3917/lautr.033.0279

Horney, K. (1926). La fuite devant la féminité. Dans Le complexe de castration, un fantasme originaire (p. 219-234). Paris: Tchou, 1978.

Iddan, C. (2017). Pousse-à-l’homme. Mental, 35 («Signes discrets dans les psychoses ordinaires»), 161-174.

Irigaray, L. (1974). Speculum de l’autre femme. Paris: Les Éditions de Minuit, 1998.

Jean, T. (1997). Considérations sur un cas de transsexualisme féminin. Journal français de psychiatrie, 5 ( «Le transsexualisme»), 29-30.

Jones, E. (1935). Sexualité féminine primitive. Dans Théorie et pratique de la psychanalyse (p. 442-452). Paris: Payot, 1969.

Klotz, H. P. (1981). Le transsexualisme, problème médical. Ornicar, 22-23, 189-211.

Kress-Rosen, N. (1999). Du côté de l'hystérie. Strasbourg: Arcanes.

Lacan, J. (1966). «Propos directifs pour un Congrès sur la sexualité féminine». Dans Écrits. Paris: Le Seuil.

Lacan, J. (1971-72). Le séminaire. Livre XIX... ou pire. Paris: Le Seuil.

Lacan, J. (1972). «L'étourdit». Dans Autres écrits. Paris: Le Seuil.

Lacan, J. (1972-73). Le séminaire. Livre XX: Encore. Paris: Le Seuil.

Maleval, J. C. (2014). Du fantasme de changement de sexe au sinthome transsexuel. Accès à la psychanalyse. Bulletin de l'ACF-VLB, 6, 127-136.

Maleval, J. C. (2019). Repères pour la psychose ordinaire. Navarin: Paris. 
Miller, J.-A. (1982-1983). Du symptôme au fantasme, et retour. Repéré à http://jonathanleroy.be/wp-content/uploads/2016/01/1982-1983-Du-symptôme-au-fantasme-etretour-JA-Miller.pdf.

Miller, J.-A. (2008). Interview par Hanna Waar. Psychologies magazine, 278. Repéré à http:// ampblog2006.blogspot.com/2008/10/interview-de-jacques-alain-miller-dans.html.

Millot, C. (1981a). Un cas de transsexualisme féminin. Ornicar, 22-23, 167-172.

Millot, C. (1981 b). Transsexualisme féminin et homosexualité. Ornicar, 22-23, 173-176.

Millot, C. (1983). Horsexe. Paris: Point Hors Ligne.

Morel, G. (2000). Ambiguïtés sexuelles: sexuation et psychose. Paris: Anthropos.

Nguyên, A. (2008). Erre de l'identité - leurre identitaire. Champ lacanien, 6 (1), 171-180.

Porge, E. (2000). Entre tentative et tentation de guérir : à partir du transsexualisme. Essaim, 6 (2). doi: 10,391 7/ess.035.0007

Rassial, J. (2004). Peut-on devenir folle? Dans P. Delaroche (dir.), Peut-on devenir fou? (p. 27-38). Toulouse: Éditions Érès. doi: 10,391 7/eres.delar.2004.01.0027

Rengifo, F. (2009). D’une discordance dans les idéaux contemporains. La clinique lacanienne, 16 (2), 203-219. doi: 10,391 7/cla.016.0203

Rubin, G. (1975). Le marché aux femmes, «économie politique» du sexe et systèmes de sexe/ genre. Dans Surveiller et jouir, anthropologie politique du sexe (p. 23-82). Paris: EPEL, 2010.

Sesé-Léger, S. (1996). Remarques cliniques à propos d'un cas de transsexualisme féminin. Dans M. Czermak et H. Frignet (dir.), Sur l'identité sexuelle. A propos du transsexualisme (p. 473-488). Paris: Éditions de l'Association freudienne internationale.

Sironi, F. (2011). Psychologie(s) des transsexuels et des transgenres. Paris: Odile Jacob. 